\title{
Note préliminaire
}

\author{
INFESTATION in vitro D'HEPATOCYTES HUMAINS \\ PAR DES SPOROZOITES DE PLASMODIUM VIVAX : \\ schizogonie et libération de mérozoïtes infestants \\ pour des hématies humaines.
}

\author{
D. MAZIER*, I. LANDAU**, F. MILTGEN**, P. DRUILHE*, C. GUGUEN-GUILLOUZO***, \\ D. BACCAM**, J. BAXTER**, J. P. CHIGOT**** et M. GENTILINI*.
}

\begin{abstract}
In vitro infestation of human hepatocytes by Plasmodium vivax sporozoïtes; maturation of schizonts and release of merozoïtes whici invaded human erythrocytes.
\end{abstract}

SUMMARY. Human hepatocytes obtained by perfusion of a liver biopsy fragment, were infected in vitro with Plasmodium vivax sporozoites raised in Anopheles stephensi. On day 7 of culture, numerous hepatocytic schizonts ranging in size from 5 to $35 \mu \mathrm{m}$ were observed. The schizont maturation was complete and led to the release of invasive merozoites as shown by in vitro infestation of human red cells layered on the hepatocytes cultures.

Des hépatocytes humains ont été obtenus par perfusion enzymatique d'une biopsie de foie. Les techniques de préparation et de culture des hépatocytes ont été décrites antérieurement (Mazier et al., 1982 ; Guguen-Guillouzo et al., 1983).

Après $48 \mathrm{~h}$ de culture, ces cellules ont été infectées in vitro par des sporozoïtes de Plasmodium vivax obtenus chez Anopheles stephensi gorgés 16 jours auparavant par nutrition artificielle avec le sang d'un malade contaminé en Inde. Dans chacune des 8 boîtes de Pétri de $35 \mathrm{~mm}$ ensemencées avec $5 \times 10^{5}$ cellules hépatiques, ont été

* Département de Parasitologie et de Médecine tropicale, Groupe Hospitalier Pitié-Salpêtrière, 47 boulevard de l'Hôpital, F 75651 Paris Cedex 13.

** Laboratoire de Zoologie-Vers, associé au CNRS, Muséum National d'Histoire Naturelle, 61, rue Buffon, F 75231 Paris Cedex 05.

*** Unité de Recherches Hépatologiques - U 49, INSERM, Hôpital Pontchaillou, F 35011 Rennes.

**** Service de Chirurgie, Groupe Hospitalier Pitié-Salpêtrière, Paris.

Ce travail a pu être réalisé grâce à l'appui financier du Ministère de la Recherche et de 1'Industrie (Département Recherche en Coopération pour le développement - contrat 82 L.076o Programme Cibles cellulaires et mode d'action des molécules antiparasitaires - contrat 82 L.o 787 ) et du programme Spécial / P.N.U.D. / Banque Mondiale / O.M.S. / de Recherche et de formation concernant les Maladies Tropicales.

Accepté le 27 juin 1983 . 
déposées 10 paires de glandes salivaires disséquées aseptiquement. Dans 2 des 3 boîtes de culture fixées au 7e jour après l'infestation et colorées au Giemsa, de nombreux schizontes ont été observés en position intracytoplasmique juxta-nucléaire. De taille variable, allant de $5 \mu \mathrm{m}$ ( 5 noyaux) à $35 \mu \mathrm{m}$ ( 2 à 300 noyaux), ils ont un aspect proche de celui des Plasmodium de muridés obtenus in vitro (Lambiotte et al., 1981; Mazier et al., 1982). Leur aspect est également comparable à celui des éléments de $12,5 \mu \mathrm{m}$ à $21 \mu \mathrm{m}$ observés en 1976 par Doby et Barker.

Dans le but de déterminer de façon non équivoque si ces schizontes étaient capables de maturation complète in vitro, $10^{8}$ globules rouges humains provenant d'un malade à fort taux de réticulocytose $(30 \%)$ ont été ajoutés aux cellules hépatiques le $10^{\mathrm{e}}$ jour. Les frottis sanguins pratiqués le $12^{\mathrm{e}}$ et le $14^{\mathrm{e}}$ jours ont permis de mettre en évidence d'assez nombreuses formes en anneau et, des trophozoïtes pigmentés. Dans aucune des cultures n'ont été objectivées de formes uninucléées qui puissent être assimilées à des hypnozoïtes (Krotoski et al., 1982) mais la technique d'immunofluorescence qui est d'une grande aide pour les localiser n'a pas été pratiquée.

$\mathrm{Au}$ cours de cette expérience, la sécrétion d'albumine humaine mesurée par immunonéphélométrie (3-4 $\mu \mathrm{g} / \mathrm{ml}$ de surnageant/jour) a confirmé que les hépatocytes conservaient leurs caractères de différenciation; toutefois, rien ne permet d'affirmer que des cellules hépatiques fonctionnelles soient nécessaires pour assurer le développement complet des stades préérythrocytaires des plasmodies humaines.

On peut espérer que ce nouveau modèle in vitro permettra d'explorer les caractères biochimiques, antigéniques et la sensibilité aux drogues de ce stade peu connu d'une parasitose humaine dont le cycle demeure encore controversé.

\section{BIBLIOGRAPHIE}

DOBY J. M. et BARKER R. - Essais d'obtention in vitro des formes préérythrocytaires de Plas modium vivax en cultures de cellules hépatiques humaines inoculées par sporozoïtes. C.R. Soc Biol. Rennes, 1976, $170,661-665$.

Guguen-Guillouzo C., Baffet G., Clément B., Bègue J. M., Glaise D. and Guillouzo A. Human adult hepatocytes : isolation and maintenance at high levels of specific functions in a co-culture system. In : Isolation, characterization and use of hepatocytes, p. ro5-I Io. Robert A. Harris-Neal W. Cornell (eds). Elsevier Science Publishing Co., Inc., Amsterdam, 1983 .

Krotoski W. A., Collins W. E., Bray R. S., Garnham P. C. C., Cogswell F. B., Gwadz R. W., Killick-Kendrick R., Wolf R., Sinden R., Koontz L. C, and Stanfill P. S. - Demonstration of hypnozoites in sporozoite-transmitted Plasmodium vivax infection. Am. J. Trop. Med. Hyg., 1982, 3I, 1291-1293.

Lambiotte M., Landau I., ThierRy N., Miltgen F. - Développement de schizontes dans des hépatocytes de rat adulte en culture après infestation in vitro par des sporozoïtes de Plasmodium yoelii. C.R. Acad. S. Paris, 1981, 293, 431-433.

Mazier D., Landau I., Miltgen F., Druilhe P., Lambiotte M., Baccam D. et Gentilini M. Infestation in vitro d'hépatocytes de Thamnomys adulte par des sporozoïtes de Plasmodium yoelii : schizogonie et libération de mérozoïtes infestants. C.R. Acad. S. Paris, 1982, 294, III, 963-965. 